\title{
The Dimensions and Composition of Stereociliary Rootlets in Mammalian Cochlear Hair Cells: Comparison between High- and Low-Frequency Cells and Evidence for a Connection to the Lateral Membrane
}

\author{
David N. Furness, ${ }^{1}$ Shanthini Mahendrasingam, ${ }^{1}$ Mitsuru Ohashi, ${ }^{1}$ Robert Fettiplace, ${ }^{2}$ and Carole M. Hackney ${ }^{3}$ \\ ${ }^{1}$ Institute of Science and Technology in Medicine, Keele University, Keele, Staffordshire ST5 5BG, United Kingdom, ${ }^{2}$ Department of Physiology, University \\ of Wisconsin Medical School, Madison, Wisconsin 53706, and ${ }^{3}$ Department of Physiology, Development, and Neuroscience, University of Cambridge, \\ Cambridge CB2 3EG, United Kingdom
}

\begin{abstract}
The sensory bundle of vertebrate cochlear hair cells consists of actin-containing stereocilia that are thought to bend at their ankle during mechanical stimulation. Stereocilia have dense rootlets that extend through the ankle region to anchor them into the cuticular plate. Because this region may be important in bundle stiffness and durability during prolonged stimulation at high frequencies, we investigated the structure and dimensions of rootlets relative to the stereocilia in apical (low-frequency) and basal (high-frequency) regions of rodent cochleae using light and electron microscopy. Their composition was investigated using postembedding immunogold labeling of tropomyosin, spectrin, $\beta$-actin, $\gamma$-actin, espin, and prestin. The rootlets have a thick central core that widens at the ankle, and are embedded in a filamentous meshwork in the cuticular plate. Within a particular frequency region, rootlet length correlates with stereociliary height but between regions it changes disproportionately; apical stereocilia are, thus, approximately twice the height of basal stereocilia in equivalent rows, but rootlet lengths increase much less. Some rootlets contact the tight junctions that underlie the ends of the bundle. Rootlets contain spectrin, tropomyosin, and $\beta$ - and $\gamma$-actin, but espin was not detected; spectrin is also evident near the apical and junctional membranes, whereas prestin is confined to the basolateral membrane below the junctions. These data suggest that rootlets strengthen the ankle region to provide durability and may contact with the lateral wall either to give additional anchoring of the stereocilia or to provide a route for interactions between the bundle and the lateral wall.
\end{abstract}

Key words: cochlea; hair cells; stereocilia; stereociliary rootlets; stereociliary stiffness; cuticular plate

\section{Introduction}

The mammalian cochlea analyses sound by means of graded mechanical resonance in the basilar membrane running along the spirally wound cochlear duct. Tone stimuli generate traveling waves that peak progressively more apically along the membrane as the frequency decreases (Robles and Ruggero, 2001). Stimulus transduction is performed by the inner (IHCs) and outer hair cells (OHCs) in the organ of Corti on the basilar membrane. The hair cells have a bundle of "hairs," the stereocilia, projecting from their apices; basilar membrane motion deflects this bundle via the overlying tectorial membrane, depolarizing or hyperpolarizing the hair cell depending on the direction of deflection. Various features of the hair cells and their bundles change systematically with frequency location (Lim, 1986).

Vertebrate hair-cell stereocilia contain a core of actin fila-

Received March 17, 2008; revised May 7, 2008; accepted May 7, 2008

This work was supported by Deafness Research UK (C.M.H.), the Steenbock Professorship (R.F., S.M.), and National Institute on Deafness and Other Communication Disorders Grant R01 DC 01362 (R.F.).

Correspondence should be addressed to David N. Furness, Institute of Science and Technology in Medicine, Keele University, Keele, Staffordshire ST5 5BG, UK. E-mail: coa14@keele.ac.uk.

DOI:10.1523/JNEUROSCI.1154-08.2008

Copyright $\odot 2008$ Society for Neuroscience $\quad$ 0270-6474/08/286342-12\$15.00/0 ments forming a paracrystalline array (Tilney et al., 1980; Slepecky and Chamberlain, 1985). A dense rootlet extends partway along the core, traversing the ankle and entering the cuticular plate (Kimura, 1975; Itoh, 1982). In chick hair cells, this rootlet consists of a short dense plug and filaments that fan out into the plate in a cone (Tilney et al., 1980) but in mammals it is a longer dense rod. Evidence from turtle cochlea (Crawford and Fettiplace, 1985) and frog crista ampullaris (Flock et al., 1977) suggests that during deflection, the stereocilia pivot around the ankle region, exposing the rootlet to mechanical stress.

In addition to detecting mechanical deflections, the hair bundle may be involved directly in the "cochlear amplifier" that enhances the passive responses of the basilar membrane to increase sensitivity and frequency selectivity. The general consensus is that the amplifier is based on contraction-elongation movements of the OHCs that boost basilar membrane motion. This somatic motility is derived from voltage-dependent conformational changes in prestin molecules in the $\mathrm{OHC}$ basolateral plasma membrane that are converted to length changes (Dallos et al., 2006). The latter may involve the actin-spectrin cytoskeletal lattice (Holley and Ashmore, 1990) underlying the prestin-bearing membrane. The hair bundle may also provide active force pro- 

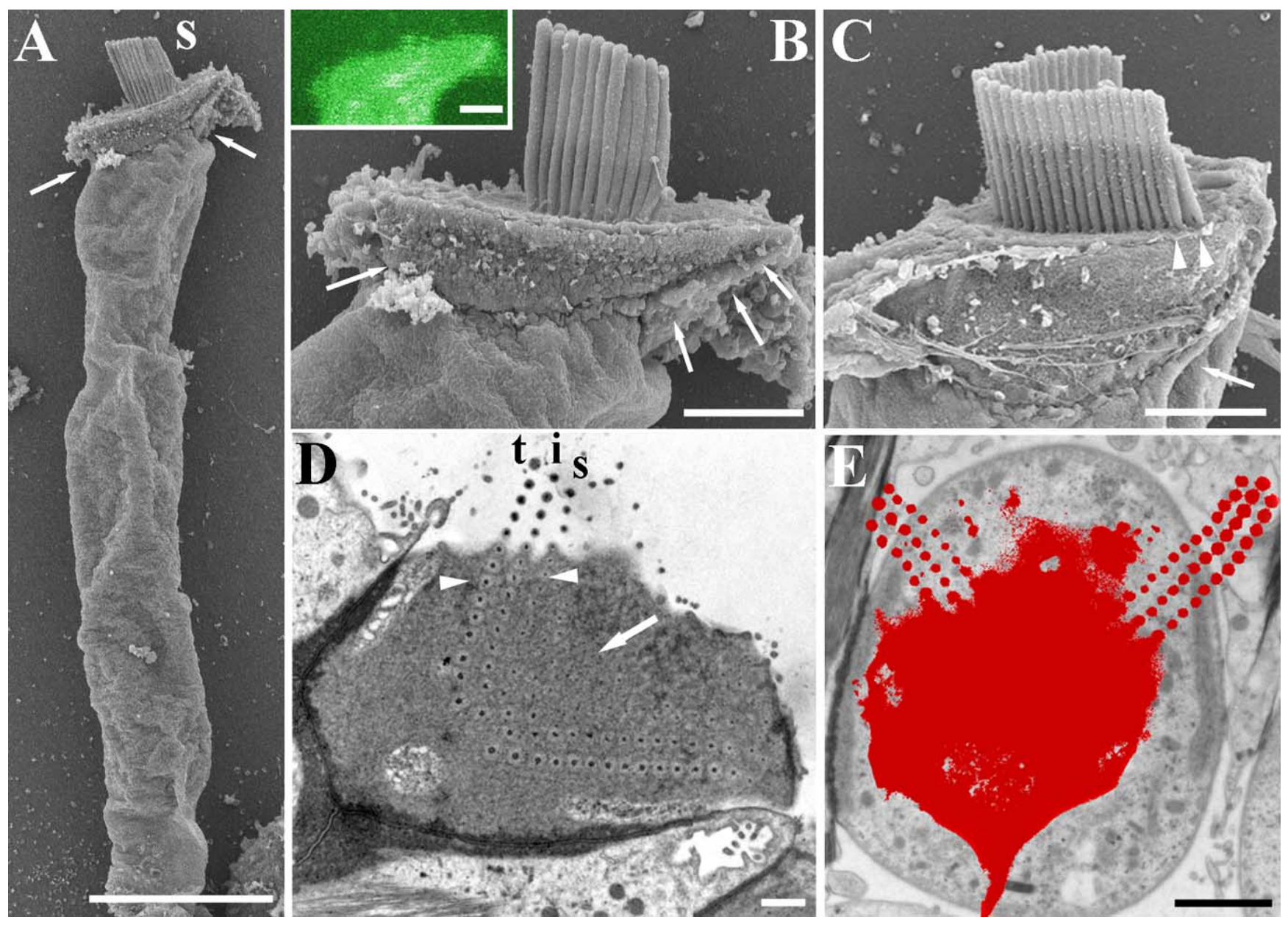

Figure 1. A, Scanning electron micrograph of an isolated guinea pig $\mathrm{OHC}$ showing the lip in the apical region (arrows). B, Detail of the lip (arrows) of the $\mathrm{OHC}$ shown in $A$. Inset, Single-plane confocal image showing the apical lip of an unfixed (living) guinea pig $\mathrm{OHC}$ after visualization with calcium green. $C$, A guinea pig basal OHC showing that the lip (arrow) is less pronounced. Arrowheads show where the stereocilia at the end of the W overhang the cell body because of the lip. $\boldsymbol{D}$, Horizontal transmission electron microscopy section of a rat $0 \mathrm{HC}$ showing the W-shape formed by the rootlets (in the center) and stereociliary ankles (at the end of one arm). Tall ( $\mathrm{t}$ ), intermediate (i), and short (s) rows can be identified with additional rootlet-like profiles inside the W where no matching stereocilia were found in serial sections (arrow). The arrowheads point to a line of three rootlets where it is possible to see them getting thinner from tall to short rows. The $W$ is not central, but lies closer to the modiolar side so that the ends of the arms are likely to overlie the lip. $E$, Outline of a horizontal section from the basal half of the cochlea through the apex and bundle (red) of a guinea pig $\mathrm{OHC}$ superimposed on the lower cell body showing how the stereocilia extend beyond the subcuticular boundaries of the cell. Scale bars: $\boldsymbol{A}, 10 \mu \mathrm{m} ; \boldsymbol{B}, \boldsymbol{C}, 2.5 \mu \mathrm{m} ; \boldsymbol{D}, 1 \mu \mathrm{m} ; \boldsymbol{E}$, $2 \mu \mathrm{m}$.

duction (Kennedy et al., 2005; Chan and Hudspeth, 2005a,b), which gives some amplification in the absence of somatic motility in prestin knock-out mouse (Liberman et al., 2004). Bundle amplification and somatic motility may potentially interact (Kennedy et al., 2006); thus, it is important to determine whether there is ultrastructural evidence for connections between the bundle and the lateral wall.

The rootlets and associated structures could influence the anchoring and flexibility of the stereocilia and potentially both their passive and active properties. We hypothesize that they may change in organization and composition tonotopically along with other gradients in hair bundles, such as stereociliary height, which decreases with frequency. To evaluate tonotopic changes, and to provide a fuller description of the rootlet system, the organization and composition of the hair-cell apex has been investigated using light, scanning electron microscopy and transmission electron microscopy of low- and high-frequency cells and immunogold studies of prestin and cytoskeletal proteins that are likely to contribute to bundle properties.

\section{Materials and Methods}

Animals and antibodies. Hearing Sprague Dawley rats [postnatal day 16 (P16) and P26], adult guinea pigs ( $>6$ weeks old), and CD-1 mice (P35) were used in this study. The primary antibodies used were monoclonal antibodies to $\beta$-actin (clone AC-15; Sigma-Aldrich) that recognize $\beta$-actin exclusively [as shown by Gimona et al. (1994)], tropomyosin (Sigma-Aldrich and Abcam; both clone TM311), and spectrin (clone MAB1622; Millipore), and polyclonal antibodies to full-length espin (a kind gift from Dr. J. R. Bartles, Northwestern University, Chicago, IL) and $\gamma$-actin (a kind gift from Dr. J. C. Bulinski, Columbia University, New York, NY). The tropomyosin antibodies label muscle cell positive controls (data not shown) and have been used to characterize tropomyosin in other tissues (Boyd et al., 1995). The spectrin antibody has been used previously to label OHC and IHC lateral walls and other structures in guinea pigs and was shown to recognize $\alpha$-spectrin in cochlear tissues by Western blotting (Mahendrasingam et al., 1998); the espin antibody labels mammalian stereocilia and recognizes the long and short isoforms of espin (Zheng et al., 2000); and the $\gamma$-actin antibody has been used previously in chick (Hofer et al., 1997) and mammalian cochlea, where it was shown to be specific to the $\gamma$-actin isoforms in cochlear tissue (Slepecky and Savage, 1994; Furness et al., 2005). Prestin antibodies were 


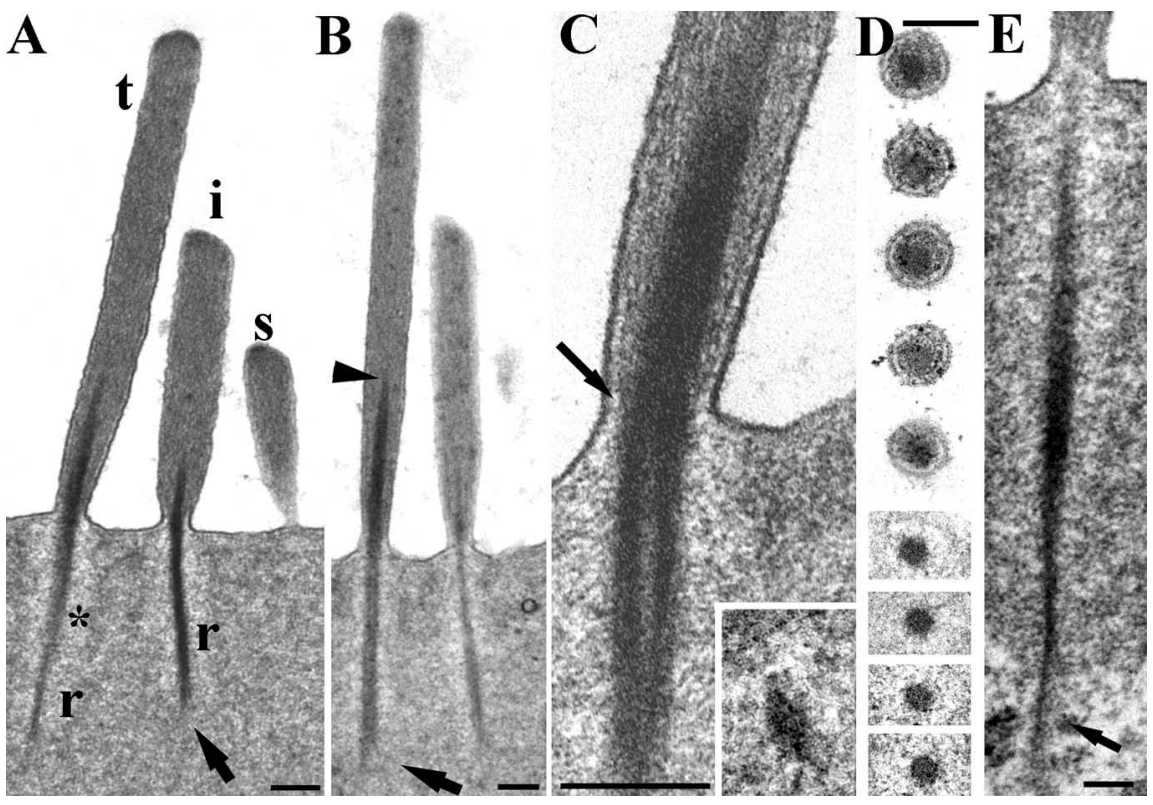

Figure 2. Structure of the rootlets in rat $\mathrm{OHCS}$. $A$, Typical example of a hair bundle in a $40 \mathrm{~nm}$ vertical ultrathin section from an apical location. The tall ( $\mathrm{t}$ ), intermediate $(\mathrm{i})$, and short $(\mathrm{s})$ stereocilia are visible and rootlets $(\mathrm{r})$ of the tall and intermediate rows; the short rootlet is out of the plane of section. A lighter zone is apparent around the rootlet (asterisk) and splaying is visible at the lower end of the intermediate rootlet (arrow). B, Similar view in a semithin $(250 \mathrm{~nm})$ section which has captured the full width and length of the rootlets (as determined from adjacent serial sections). Note the tapering of the rootlet into the shaft, particularly of the tall stereocilium (arrowhead), and the splaying of the end of the tallest stereociliary rootlet in the cuticular plate (arrow). $\boldsymbol{C}$, The ankle region of a tall stereocilium, showing the filaments in the stereocilium shaft converging onto the rootlet (arrow shows this on one side). Inset, A cross section of a rootlet deep in the cuticular plate showing the crescent shaped profile. D, Serial sections of a single stereocilium through the ankle region. The rootlet is the dense central material. $\boldsymbol{E}$, Example of a rootlet extending through the lower edge of the cuticular plate (arrow) into the apical cytoplasm, where it splays out. Scale bars: $A-C$, inset, $200 \mathrm{~nm}$; $D, 100 \mathrm{~nm} ; E, 150 \mathrm{~nm}$.

made to the C-terminal sequence (CTVLDPQEDMEPNATPTTPEA) by Bethyl Laboratories and have not been tested by Western blotting, but specifically label known prestin-bearing membranes in wild-type mice and not in prestin knock-out mice (tissue kindly supplied by M. A. Cheatham and P. Dallos, Northwestern University, Chicago, IL). The labeling is abolished by preadsorption with the antigen (data not shown). The secondary antibodies were goat anti-rabbit and anti-mouse IgGs conjugated to various-sized gold particles (British BioCell).

All animals were treated in accordance with the United Kingdom Animals (Scientific Procedures) Act of 1986, and all procedures were approved either by the ethical committee of Keele University or the animal care committee of the University of Wisconsin.

Preparation of isolated hair cells for in vitro and scanning electron microscopic observation. Hair cells were mechanically isolated from the guinea pig organ of Corti and prepared for observation both by light microscopy and confocal imaging of living cells and for scanning electron microscopy. The purpose of these procedures was to provide an overview of the shape of the cochlear apex in isolation from the surrounding cells, within which context the ultrastructural observations could be better interpreted.

Animals were given an overdose of sodium pentobarbitone (i.p.; Pentoject; Animalcare), decapitated, and the bullae removed and opened in HBSS comprising (in mM) $137 \mathrm{NaCl}, 0.7 \mathrm{NaH}_{2} \mathrm{PO}_{4}, 5.8 \mathrm{KCl}, 1.3 \mathrm{CaCl}_{2}$, $0.9 \mathrm{MgCl}_{2}, 10 \mathrm{HEPES}$, and $5.6 \mathrm{D}$-glucose. The thin exposed bony shell of the cochlea was then removed and the modiolus broken across at the base so that the central spiral on which the organ of Corti is situated could be taken out. The organ of Corti was then unwound by gripping the spiral limbus in the basal turn and peeling it from the osseous spiral lamina in an apical direction. The segment(s) of organ of Corti were then repeatedly aspirated through a $100 \mu \mathrm{l}$ disposable pipette tip onto a poly-Llysine-coated coverslip and allowed to settle for $5 \mathrm{~min}$.

For confocal observations of unfixed cells, the covering volume of HBSS was removed until only a thin layer remained and replaced by a covering volume of HBSS containing $5 \mu \mathrm{M} \mathrm{cal-}$ cium green AM (Invitrogen) for $\sim 5 \mathrm{~min}$. After washing, the cells were examined in a Bio-Rad MRC-1024 confocal system using the kryptonargon laser with excitation at $488 \mathrm{~nm}$ and emission filters of 522/35 nm.

For scanning electron microscopy, the HBSS was removed as before and replaced by $2.5 \%$ glutaraldehyde in $0.1 \mathrm{M}$ sodium cacodylate buffer containing $2 \mathrm{mM} \mathrm{CaCl}_{2}$ for $1 \mathrm{~h}$. After washing in the same buffer, the samples were dehydrated in an ethanol series and criticalpoint dried using $\mathrm{CO}_{2}$ as the transitional fluid. The coverslips were then attached to stubs with adhesive carbon pads, and the samples were coated with gold for $2 \mathrm{~min}$ at $25 \mathrm{~mA}$ in an Emscope SB 250 sputter coater.

Fixation of tissue and resin embedding for transmission electron microscopy. For conventional electron microscopy and serial section reconstruction, cochleae were obtained as above and fixed by intralabyrinthine perfusion, either with $2.5 \%$ glutaraldehyde alone in $0.1 \mathrm{~m}$ sodium cacodylate $/ 2 \mathrm{~mm}$ calcium chloride buffer, $\mathrm{pH}$ 7.4 , or with the addition of $1 \%$ tannic acid to emphasize filamentous structures. They were immersed in fixative for $2 \mathrm{~h}$ then postfixed in $1 \%$ osmium tetroxide for $1 \mathrm{~h}$, washed, and dehydrated in a graded series of ethanols. The larger cochleae of guinea pig and rats were dissected into segments, whereas the smaller cochleae of mice were decalcified in 5 mM EDTA for $3 \mathrm{~d}$ and left whole. They were then embedded in Spurr resin (Agar Scientific) according to the method described by Furness and Hackney (1985).

For postembedding immunocytochemistry, cochleae were perfused with $4 \%$ paraformaldehyde and $0.1 \%$ glutaraldehyde in $0.1 \mathrm{~m}$ phosphate buffer $(\mathrm{PB})$ at $\mathrm{pH} 7.4$ through the round window and a small hole in the apex and then immersed in the same fixative for $2 \mathrm{~h}$. After washing in $0.01 \mathrm{M}$ PBS, pH 7.4, the bony walls of the cochleae were partially removed and the cochleae were either dissected into segments or embedded whole.

Cochleae and segments were embedded either in LR White (Agar Scientific) or Lowicryl HM20 resin (Agar Scientific). For LR White embedding, they were dehydrated in a graded series of ethanols, infiltrated in resin at room temperature for $16 \mathrm{~h}$, and embedded in pure resin polymerized at $50^{\circ} \mathrm{C}$ for $24 \mathrm{~h}$ in gelatin capsules according to the method described by Mahendrasingam et al. (1998). For Lowicryl embedding, fixed cochlear segments were cryoprotected by immersion in increasing concentrations of glycerol in PBS (10, 20, and 30\%), then plunge frozen in liquid propane and immersed in anhydrous methanol containing $0.5 \%$ uranyl acetate at $-85^{\circ} \mathrm{C}$, brought gradually up to $-45^{\circ} \mathrm{C}$, rinsed in anhydrous methanol, and infiltrated with increasing concentrations of Lowicryl HM20 resin according to the method described by Furness and Lehre (1997). These samples were polymerized with UV light and allowed to warm up to $20^{\circ} \mathrm{C}$ over $33 \mathrm{~h}$.

For whole cochleae embedded in either Spurr or LR White resin, samples were microsliced to obtain segments of the organ of Corti using a Malvern 2A Microslicer with an annular diamond blade as described by Jiang et al. (1993). For nonserial sectioning and qualitative ultrastructural observations, $40-100 \mathrm{~nm}$ sections were cut on a Reichert Ultracut microtome, mounted on 200-mesh thin bar copper grids, and stained with $2 \%$ ethanolic uranyl acetate for $20 \mathrm{~min}$ and $2 \%$ lead citrate for $2-5$ $\min$.

Serial section reconstruction. To better determine the relationship between stereocilia and their rootlets, and to understand the substructure of the cuticular plate, serial sections were taken and three-dimensional (3D) reconstructions were performed. Sections were cut in two planes, 

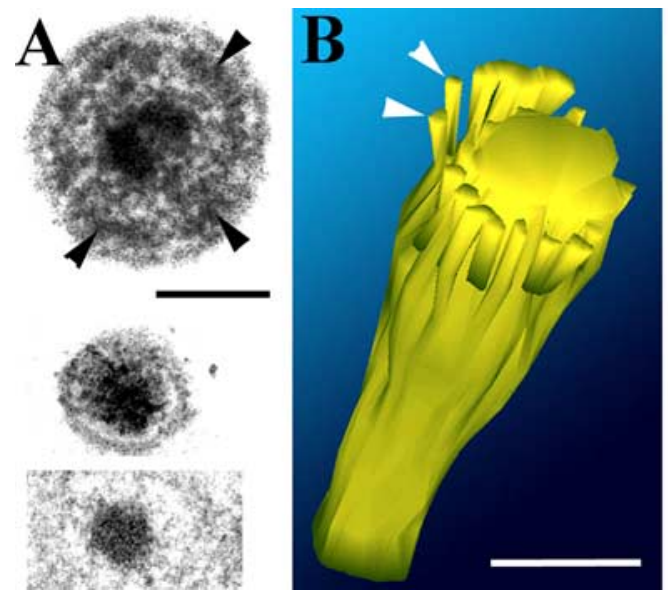

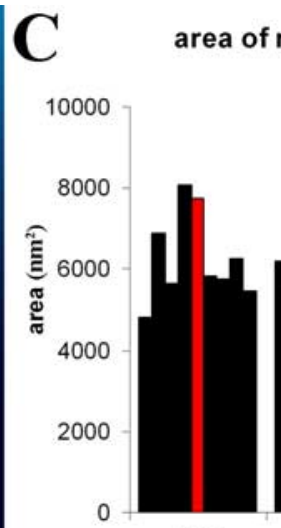

Tall 1 rea of rootlet profiles in serial sections



Figure 3. A, Top, Cross section of a guinea pig tall stereocilium showing the central core of the rootlet and a peripheral dense ring around it (arrowheads). Bottom two panels show two sections of a rat $\mathrm{OHC}$ stereocilium from a series used to create the $3 \mathrm{D}$ reconstruction in $\boldsymbol{B}$. The top of the two is at the level of the ankle region, which is virtually filled with dense material. The bottom of the two is at the level of the cuticular plate, showing that the rootlet has narrowed. Scale bar, $100 \mathrm{~nm} . \boldsymbol{B}, 3 \mathrm{D}$ reconstruction of the rootlet in the ankle region. The central core is joined by peripheral dense filaments of material (e.g., arrowheads) that may coat converging actin filaments. These sheaths around the filaments end above the level of the apical membrane. Scale bar, $100 \mathrm{~nm}$. $\boldsymbol{C}$, Cross sectional area in nanometers squared of the rootlet material in five different sets of cross sections spanning $\sim 900 \mathrm{~nm}$ through the ankle region of two tall, two intermediate, and one short stereocilium from a rat $\mathrm{OHC}$. Each section is represented by a single black bar except for the section representing the upper surface of the cuticular plate, shown in red. The thickest part of the rootlet is usually just at the entry into the apical membrane and the cross sectional area decreases across the tall to short rows.
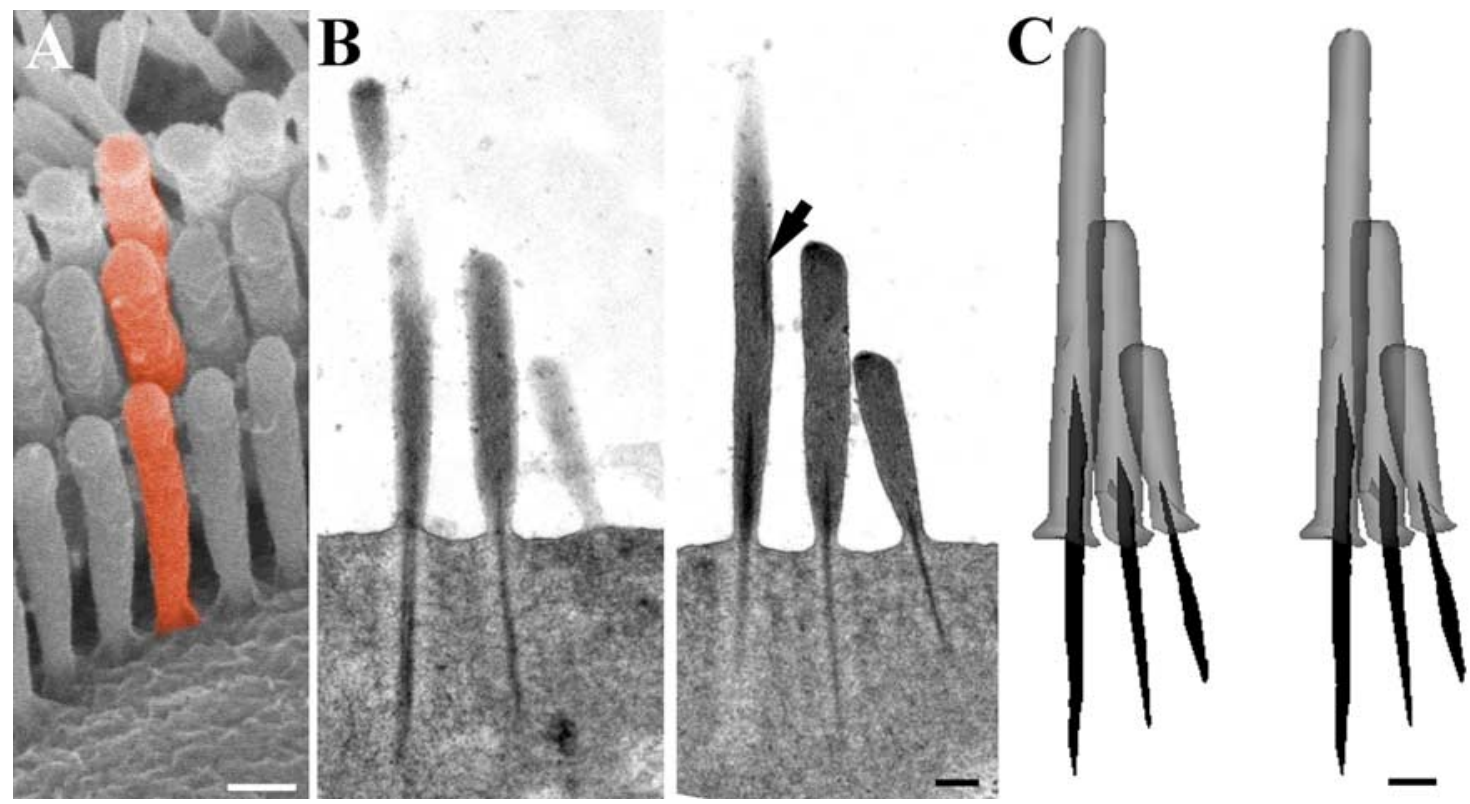

Figure 4. Serial section reconstruction of representative stereocilia from an apical hair bundle. A, Scanning electron micrograph of a guinea pig OHC bundle to illustrate the concept of a "column" of stereocilia (colored red). $\boldsymbol{B}$, Two sequential sections of the nine semithin $(200 \mathrm{~nm})$ serial sections of a rat $\mathrm{OHC}$ used to construct a column of stereocilia as illustrated in $\boldsymbol{A}$. Note the dense rootlet like material in the upper region of the tall stereocilium displaced toward the edge (arrow). This is discontinuous with the normal rootlet material. C, Stereopair of the reconstructed column. Note how the thickness and length of the rootlet is approximately proportional to the height of the stereocilia in each row. The upper dense material has been omitted for clarity. Scale bars, $200 \mathrm{~nm}$.

either radially across the cochlear duct (hereafter referred to as vertical with respect to stereocilia) or parallel to the surface of the organ of Corti (horizontal with respect to the stereocilia). Serial ultrathin $(50-100 \mathrm{~nm}$ ) or semithin (150-250 nm) sections were collected onto Formvar-coated copper slot grids. Serial sections of the rootlets and cuticular plate were imaged using a JEOL 1230 transmission electron microscope and images were acquired with a Megaview III digital camera and analysSIS software (Olympus Soft Imaging Systems). Reconstructions were made using Reconstruct software authored by J. C. Fiala (Boston University, Boston, MA), freely available at http://www.bu.edu/neural/Reconstruct.html.

Stereociliary and rootlet dimensions were determined from serial sections. In favorable vertical orientations, this was done by superimposing the sections in Adobe Photoshop and increasing layer transparency to overlie contiguous regions so that the full length of the rootlet and stereocilium were measured. In less favorable section planes, full 3D reconstruction was required using the software indicated above. Because the stereocilia and their rootlets were longer in apical regions and the bundles more prone to disarray, it was not always possible to obtain a full-length reconstruction of both together. Thus, to increase the data set, some measurements were made of stereocilia independently of rootlets and vice versa.

Postembedding immunogold labeling. Ultrathin sections (100-120 nm) were cut and collected on 200-mesh nickel grids coated with glue (Gluepen; Agar Scientific). Sections were blocked in 10-20\% goat serum in $0.05 \mathrm{~m}$ Tris-buffered saline (TBS, pH 7.4) (GS-TBS) and incubated overnight at $4^{\circ} \mathrm{C}$, or for $2 \mathrm{~h}$ at $37^{\circ} \mathrm{C}$, in primary antibodies to $\beta$-actin 
$(1: 200-1: 20,000), \quad \gamma$-actin $(1: 1000-1: 12,000)$, tropomyosin (1:25), spectrin (1:10), prestin (1: 5000 ), or espin (1:5), all diluted in $1 \%$ bovine serum albumin (BSA) in $0.05 \mathrm{~m}$ TBS (BSATBS), washed in BSA-TBS, and blocked in 10$20 \%$ GS-TBS followed by appropriate secondary antibody conjugated to 10 or $15 \mathrm{~nm}$ gold particles diluted 1:20 in 1\% BSA-TBS for $2 \mathrm{~h}$ at room temperature. For double labeling, sections were incubated in a mixture of primary antibodies to $\gamma$-actin $(1: 1000)$ and $\beta$-actin (1: 1000 ) followed by a mixture of goat anti-rabbit IgG $30 \mathrm{~nm}$ gold conjugate and goat anti-mouse IgG 15 or $10 \mathrm{~nm}$ gold conjugate (1:20). Immunogold-labeled sections were then stained in $2 \%$ uranyl acetate and examined using a JEOL 100-CX or JEOL 1230 transmission electron microscope, with images acquired as before using the Megaview III digital camera system. For negative controls, primary antibodies were replaced with the dilution buffer.

\section{Results}

\section{Morphology of the apex and position of} the stereociliary bundle

To observe the morphology of the apical region free from other anatomical structures within the cytoarchitecturally complex reticular lamina (surface of the organ of Corti), OHCs were isolated and examined both in fresh preparations in vitro or fixed for scanning electron microscopy observation. Although isolation can cause cells to be disturbed or swollen, only those that retained a normal cylindrical shape as seen in rapidly fixed preparations were examined.

In both unfixed and fixed guinea pig OHCs, the long axis of the cell body can lie at an angle to the apical surface (Fig. $1 A$ ) and there is a widening at the apex that gives a pronounced lip containing the edges of the cuticular plate (Fig. $1 B$ ). The lip is less pronounced in shorter, probably basal hair cells (Fig. 1C). The W-shaped bundle tends to be situated "forward" (toward the modiolus) relative to the central long axis of the cell, most prominently in guinea pig apical hair cells (Fig. 1D). This position results in the stereocilia at one or both ends of the arms of the $\mathrm{W}$ overlying the lip beyond the edges of the cell body as it narrows below the apex (Fig. 1E).

\section{Ultrastructure of the rootlets}

Typically, there are three rows of stereocilia (tall, intermediate, and short) in OHCs, which, in our fixed material, converge distally, with the tallest and intermediate rows angled slightly forward (i.e., in the negative direction with respect to the excitatory bundle stimulus). Stereocilia of all three rows have a rootlet (Figs. $1 D, 2 A)$, the main portion of which consists of an electron-dense accumulation of material associated with actin filaments starting some distance up the core of the stereocilium and extending down through the ankle region into the cuticular plate (Fig. $2 A, B)$. In all of the rodents observed, the length of this rootlet is greatest for the tallest stereocilia and least for the shortest. A slight bend can occur in the rootlet where it enters the cuticular plate; a negative tilt of the stereocilium appears to result in the portion of the rootlet above the ankle tilting the same way (Fig. 2A).

Within the stereocilium itself, the rootlet material extends upward, tapering gradually to a point typically about one-third of
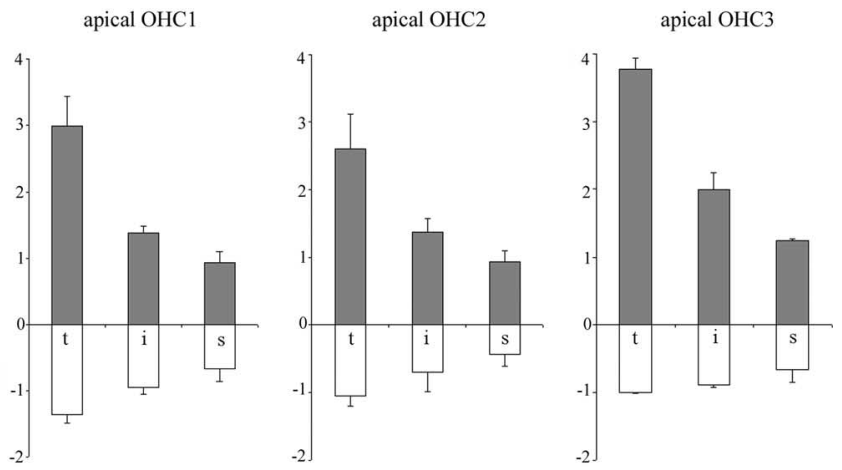

basal $\mathrm{OHC} 2$

basal $\mathrm{OHC} 3$
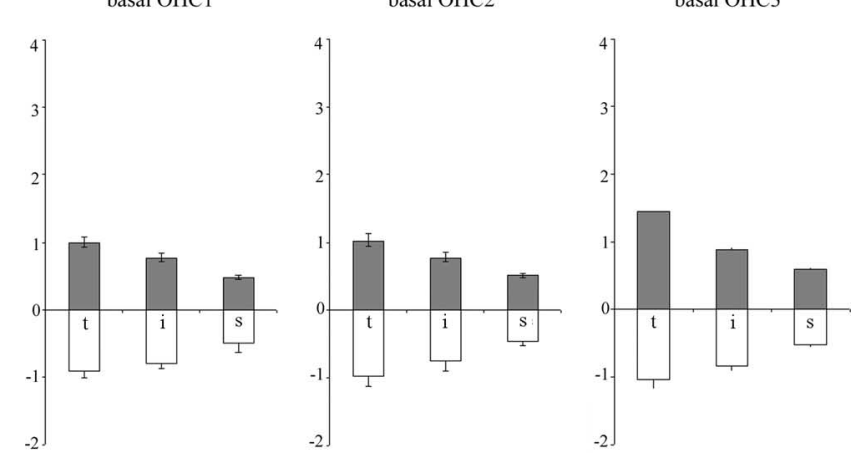

Figure 5. Histogram showing the mean lengths of stereocilia and cuticular portion of the rootlets from apical and basal hair to a much lesser degree. Data for IHCs derived from one animal are shown for comparison and reveal a similar pattern. t, Tall; i, intermediate; s, short; gray upward bars are the stereocilia; white downward bars are the rootlets.

the way along the shaft in apical hair bundles (Fig. $2 B$ ) and about one-half in basal OHCs (data not shown). Above the level of the apical membrane, peripheral actin filaments of the stereocilium shaft curve to contact the central rootlet material rather than terminating on or near the membrane (Fig. $2 C$ ). Horizontal sections through the cuticular plate show that the rootlets of all three rows of stereocilia continue in rows maintaining their general position relative to adjacent rootlets (Fig. $1 D$ ). There are also additional rootlet-like profiles corresponding to microvilli or stereocilia that may have withdrawn during development (Fig. 1D).

By following serial horizontal sections of individual rootlets (Figs. 2D, 3A), it is possible to show that as the rootlet progresses down through the ankle region of the stereocilium, it consists of a central core surrounded by a concentric dense ring associated with the peripheral actin filaments (Fig. 3A). This dense ring narrows toward the central core of rootlet material and converges on it together with the peripheral actin filaments. This has been observed in all three rodent species studied (rats, mice, and guinea pigs) and has been reconstructed from horizontal serial sections of rat hair cells using 3D-reconstruction software (Fig. $3 B)$. The reconstruction shows that this material forms or coats the filaments, which converge onto and coalesce with the core near the entry through the apical surface.

Measurements of the relative cross-sectional area of the central rootlet core in serial sections from apical rat $\mathrm{OHCs}$ indicate that the rootlet is widest just above the point of entry, where it is $\sim 100 \mathrm{~nm}$ in diameter (Fig. 3C). The cross-sectional area also decreases across the three rows from tall to short (Figs. $1 D, 3 C$ ). Deeper into the cuticular plate the rootlets of taller and intermediate stereocilia in particular may become hollow in appearance, 
Table 1. Summary of various parameters of stereocilia and rootlet lengths in rat cochlea

\begin{tabular}{|c|c|c|c|c|}
\hline St/rt type & Apical (A) & Basal (B) & $p(A$ vs $B)$ & Ratio A/B \\
\hline Tall st & $2.99 \pm 0.70(39)$ & $1.06 \pm 0.09(28)$ & $>0.001$ & 2.82 \\
\hline Tall rt & $1.13 \pm 0.20(32)$ & $0.96 \pm 0.14(28)$ & $>0.001$ & 1.2 \\
\hline Ratio tall st/rt & 2.65 & 1.1 & & \\
\hline Intermediate st & $1.56 \pm 0.31(34)$ & $0.80 \pm 0.08(29)$ & $>0.001$ & 1.95 \\
\hline Intermediate rt & $0.80 \pm 0.25(31)$ & $0.79 \pm 0.12(31)$ & 0.41 & 1 \\
\hline Ratio intermediate st/rt & 1.95 & 1 & & \\
\hline Short st & $0.97 \pm 0.17(23)$ & $0.52 \pm 0.05(35)$ & $>0.001$ & 1.87 \\
\hline Short rt & $0.57 \pm 0.2(22)$ & $0.48 \pm 0.09(26)$ & 0.047 & 1.2 \\
\hline Ratio short st/rt & 1.7 & 1.1 & & \\
\hline
\end{tabular}

The means \pm SDs of the height of stereocilia (st) and length of rootlet (rt) are shown for apical and basal locations for each stereociliary row in all OHCS analyzed in the present study. (Values in parentheses indicate the total number of stereocilia measured in each category using all three measurement techniques described in Materials and Methods.) Column $p$ indicates the probability of a significant difference between apical and basal (A vs B) data determined using a one-tailed $t$ test. The ratio of apical to basal values is also given, in the column labeled Ratio (A/B). The ratio of stereocilia to relevant rootlet length (st/rt) is given in the row beneath each set of length/height data.
This material is often displaced to the periphery of the core (Fig. $4 B$ ).

These ultrastructural features of the rootlet were observed in both apical and basal locations, although primarily measured from apical hair cells. The structure of the rootlets was similar in both locations. However, the fact that the rootlets change width and length with the different rows of stereocilia suggested that their dimensions may differ in different locations along with the height of the stereocilia. The dimensions relative to stereociliary height were thus explored.

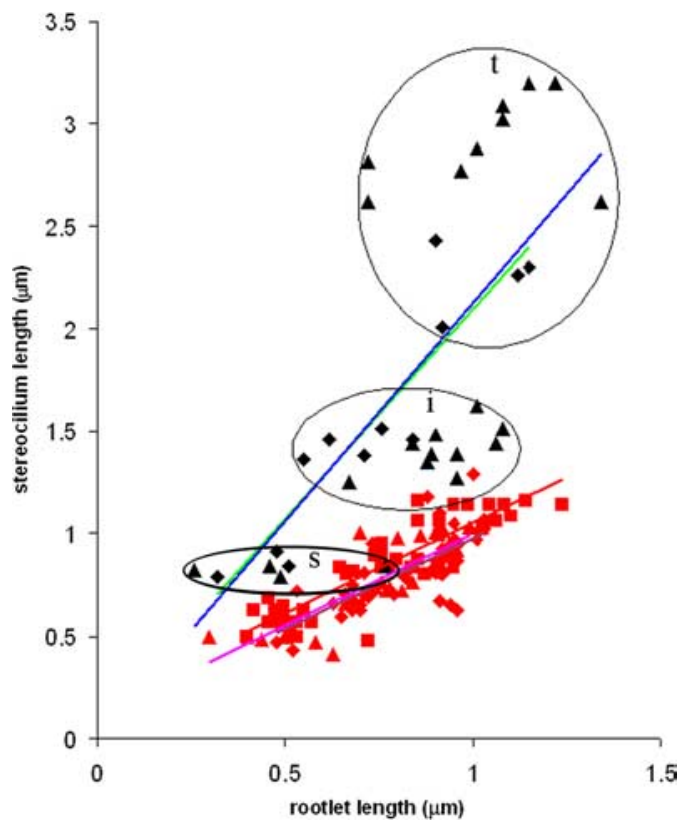

Figure 6. Scatterplot of all of the complete stereocilia-rootlet pairs measured in this study from the rat cochleae. Apical data from two of the three animals (denoted by diamonds or triangles) are plotted with black symbols and blue and green trend lines respectively; basal data from the same two animals (denoted again by diamonds, triangles) are plotted as red symbols with pink and purple trend lines respectively. Additional data from a third animal for the basal region are plotted in red squares with a red trend line. In both apical and basal data, the height of stereocilia and length of rootlets are correlated, as indicated by the trend lines. However, the slopes of the trend lines for the basal data are shallower than for the apical data. The three different rows of stereocilia-rootlet pairs are not easily distinguishable in basal data when measurements from all animals are plotted on the same graph, because they partially overlap, but can be clearly seen in the apical data where they are circled and labeled. $t$, Tall; i, intermediate; 5 , short.

and deeper still, they often become thin and flattened or crescent shaped (Fig. 2C, inset). It is not possible to determine in our material whether some of the actin filaments in the shaft of the stereocilium above the cuticular plate extend down with the dense material or whether new filaments are present in the rootlet below the ankle region. In longitudinal section, the rootlets tend to remain relatively rod-like until their lowest end where they can splay out (Fig. $2 A, B$ ), with dense material becoming reduced and individual or bundles of filaments diverging from the core.

Rootlets sometimes extend beyond the cuticular plate (Fig. $2 E)$, where this splaying can also occur in all three species. In some cases, dense material is also found further up the shaft appearing similar to rootlet material but disconnected from it.

\section{Measurement of stereocilia height and rootlet lengths}

To determine more accurately how the rootlet length corresponds with the height of stereocilia within a bundle and in different frequency locations, reconstructions were made from serial vertical $0.2-0.25 \mu \mathrm{m}$ sections (Fig. 4). By reconstructing columns of stereocilia (as illustrated in Fig. 4A), it was confirmed that within a given hair cell, the lengths of the rootlets approximately correlates to the length of the stereocilium (i.e., shorter rootlets are found projecting from shorter stereocilia). However, it was apparent when comparing apical and basal reconstructions that the rootlet length changes much less for each equivalent row

To quantify this, measurements were made from the rat cochlea of stereociliary height and the length of the rootlet within the cuticular plate in apical (80\% distance from base corresponding to $\sim 4 \mathrm{kHz}$ ) and basal (20\% distance corresponding to $\sim 30$ $\mathrm{kHz}$ ) locations (Fig. 5, Table 1). Plots of the average profile of hair bundles from IHCs and OHCs in the three separate rows are shown based on all measurements obtained from three animals (Fig. 5). These show that for all three rows of OHCs the rootlets are shorter for shorter stereocilia, as noted above, but that they do not change proportionately with stereocilium height when comparing different locations. Table 1 shows all of the data for $\mathrm{OHCs}$ grouped together. In OHCs from the apical low-frequency location, the tallest stereocilia were $\sim 3 \mu \mathrm{m}$ high and had rootlets that were $1.13 \mu \mathrm{m}$ long, a ratio of 2.65:1; intermediate stereocilia were $1.56 \mu \mathrm{m}$ tall and their rootlets were $0.8 \mu \mathrm{m}$, a ratio of $\sim 1.9: 1$; and the shorter stereocilia were $0.97 \mu \mathrm{m}$ with $0.57 \mu \mathrm{m}$ rootlets, a ratio

In basal high-frequency OHCs, the stereocilia were approximately one-half or less of the height of the equivalent row in the apical high-frequency location (Table 1). The differences in stereociliary height for each equivalent row were significant (Student's one-tailed $t$ test; $p>0.001$ ). In OHCs from the basal highfrequency location, the tallest stereocilia were $1.06 \mu \mathrm{m}$ high and had rootlets that were $0.96 \mu \mathrm{m}$ long, a ratio of $\sim 1.1: 1$; intermediate stereocilia were $0.80 \mu \mathrm{m}$ and their rootlets were $0.79 \mu \mathrm{m}$, a ratio of $\sim 1: 1$; and the shorter stereocilia were $0.52 \mu \mathrm{m}$ with 0.48 $\mu \mathrm{m}$ rootlets, a ratio also of $\sim 1: 1$ (Table 1 ). Thus, in basal region, the rootlets were approximately the same length as their respective stereocilia whereas in the apical region they were approximately one-half the length. Statistical comparison showed that both the tall and the short rootlet lengths were significantly different between apical and basal locations $(p>0.001$ and $p=$ 0.047 , respectively), whereas the intermediate rootlet length was not.

This difference in relationship between the stereocilia and than the stereociliary height. of 1.7:1. 
their rootlets in the two locations is also illustrated in Figure 6. Here, stereociliary height is shown plotted against rootlet length for complete stereocilia-rootlet pairs (i.e., where both could be measured together) for apical region (two animals) and basal region (three animals). Within a given location, the stereocilium height and rootlet length are correlated. However, the slope of the relationship differs being shallower in the basal location compared with the apical location. This confirms that there is a disproportionate change in rootlet length with location compared with stereocilium height.

Preliminary data were also obtained from rat IHCs (Fig. 5). These also showed that stereocilia and rootlets were shorter in basal locations but not proportionately, the rootlets changing less than the stereocilia. In both locations it was noticeable that, although the height of the tallest IHC stereocilia was greater overall, the rootlets were similar in length to those of the tallest stereocilia of OHCs from the same location.

\section{Rootlet connections to the lateral wall at the junctional complex}

A particularly striking feature of some rootlets from all three rows of stereocilia was that they appeared to end in or close to the dense material of the lateral wall at the junctional complex. This was noted in both apical (Fig. 7A) and basal locations (Fig. $7 B, C$ ) in all three species. For the taller stereocilia, this connection could occur in the lower half of the junctional complex region. In horizontal serial sections from both rat (Fig. 7D) and guinea pig, there was evidence of a close approach of rootlets to the edge of the cell by the wings of the bundles on both sides.

The structure of these connections was examined in all three rodent species. The apparent connections appeared to be represented by direct continuity between the dense material in the junction and the rootlet, and in some places, fine filaments also extended out from the rootlet into the former (Fig. $7 E, F$ ).

These rootlet-lateral wall connections occur mainly because of the shallow lip around the apical portion of the OHC, which appears to contain the periphery of the cuticular plate. In the region of the lip, the rootlets, as elsewhere, progress vertically without substantially bending within the cuticular plate and their length is sufficient, together with the shallow depth of the lip, to encounter the lateral wall.

\section{Substructure of the cuticular plate}

Within the cuticular plate, a network composed of both dense and filamentous material forms a mesh around the rootlets in all three rodents. The appearance of the substructure of this meshwork is affected by the thickness of sections used to examine it. In $100 \mathrm{~nm}$ ultrathin sections, the dense meshwork stands out more clearly and produces the appearance of a zone of lighter material around the rootlet; however, in terms of its electron density, this zone has similar density to portions of the mesh-free matrix of the plate (Fig. $8 \mathrm{~A}$ ). In very thin $(40 \mathrm{~nm})$ sections, the dense material is difficult to see; however, horizontally and vertically (Fig. $8 \mathrm{~B}$ ) aligned filaments can be seen in the cuticular plate between rootlets. A distinct submembrane layer sits on top of the cuticular plate and meshwork and comes into lateral contact with rootlets just below their entry into the cell (Fig. 8C).

The meshwork occurs in the upper part of the cuticular plate and its depth varies between species. In rat OHCs, the denser parts of the mesh occupy a relatively narrow region below the apical membrane, whereas less dense vertical portions extend down between rootlets in the region of and probably associate with the vertically aligned filaments noted above (Fig. $8 D$ ).

The meshwork was reconstructed from 10 serial $100 \mathrm{~nm}$ ultrathin sections of guinea pig OHCs (Fig. 8E,F) and a similar structure was confirmed qualitatively in serial sections of rat hair cells. The meshwork surrounds each rootlet forming a thick sheath, which is particularly prominent around the rootlets of the taller and intermediate stereocilia. Ultrastructurally, the sheath is in places connected to the rootlet by very fine filaments. The meshwork extends across the full extent of the cuticular plate. The cytoplasmic channels that occur in the periphery of the plate (Fig. $8 A$ ) were also reconstructed (Fig. $8 E$ ). The main channel on the strial side of the hair cell contains the basal body and reaches to the surface of the cell, as do some of the lateral channels. There 

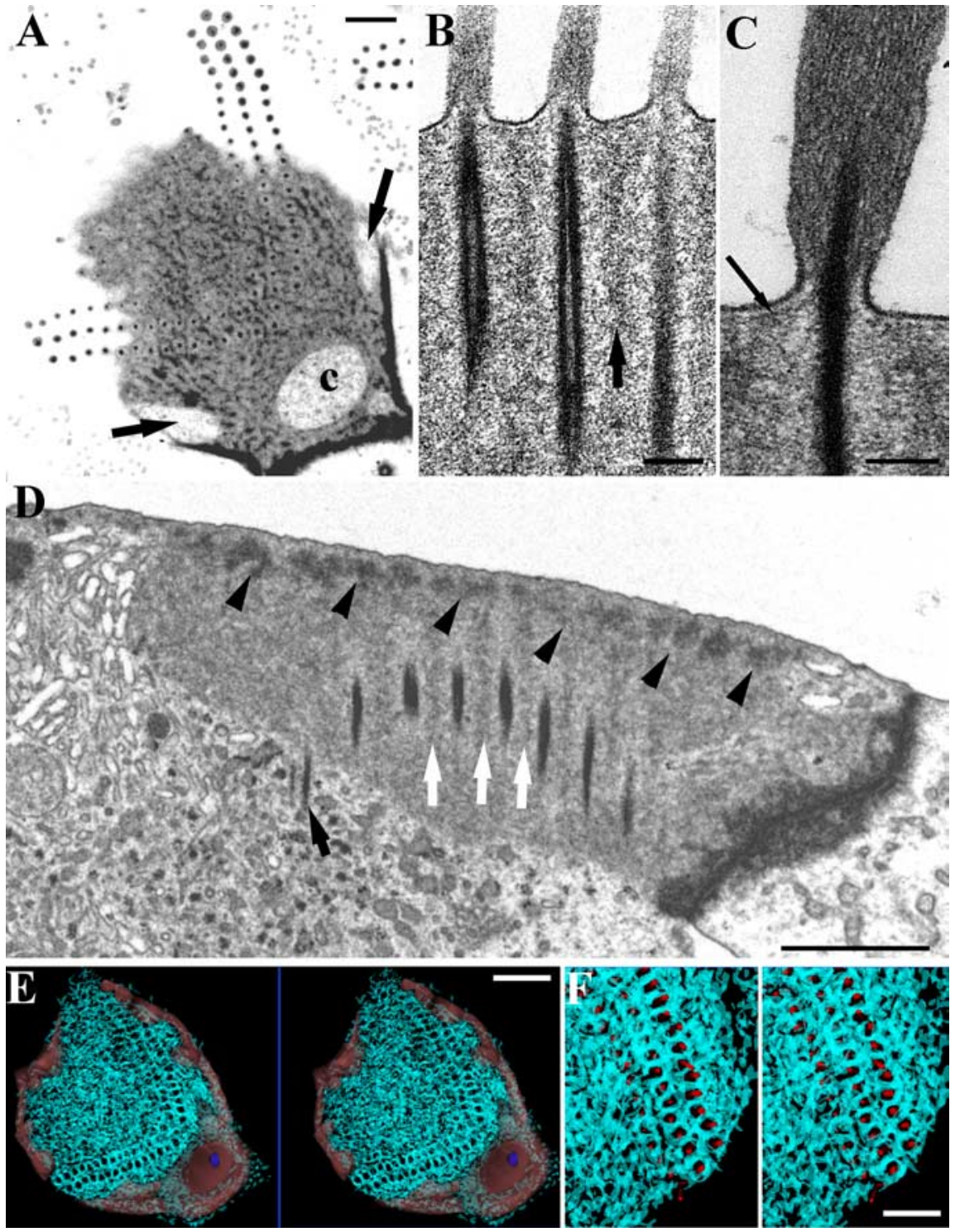

Figure 8. The structure of the meshwork in the cuticular plate. $A$, Horizontal section of a guinea pig $\mathrm{OHC}$. The dense material forms a mesh around the rootlets extending over the superficial aspect of the plate. Channels in the periphery of the cuticular plate (arrows) and at the apex of the $\mathrm{W}$ (c) are visible. $\boldsymbol{B}$, Vertical section of a rat $\mathrm{OHC}$, showing vertically orientated parallel actin filaments between the rootlets (arrow). C, A subapical dense layer extends over the cuticular plate upper surface and contacts the rootlet where it enters the plate (arrow); rat $\mathrm{OHC}$. D, Vertical section of a rat $\mathrm{OHC}$ showing dense material in the apical meshwork along the upper edge of the plate (arrowheads). Note lighter material extending down from the mesh (white arrows) and one rootlet penetrating into the cytoplasm below the cuticular plate (black arrow). $\boldsymbol{E}, \boldsymbol{F}$, Two sets of stereopairs at low and intermediate magnification of the meshwork reconstructed from serial sections of a guinea pig $\mathrm{OHC}$ (cyan traces). The channels are indicated in light red in $E$ and the basal body (normally found in the large channel on the strial side of the bundle) is shown in blue. Reconstruction shows that the meshwork around the rootlets extends in $3 D$ so that each is ensheathed by the dense/filamentous material of the meshwork. $\boldsymbol{F}$, Rootlets are shown as red cylinders sitting in their sheaths. Scale bars: $A, D, F, 1 \mu \mathrm{m} ; \boldsymbol{B}, \boldsymbol{C}, 100 \mathrm{~nm}$; $E, 2 \mu \mathrm{m}$.

are no obvious qualitative differences in these cuticular-plate structures in different locations.

\section{Compositional aspects of the apical region}

Although much is known about the composition of the apical region, it has generally been examined with light microscopy, either immunofluorescence with a conventional epifluorescence microscope or confocal microscopy. However, this does not give the precision needed for the present study. A postembedding immunogold technique was therefore used, which has the advantage that, because labeling is performed on ultrathin sections, all areas of the cell are fully accessible and the ultrastuctural distribution can be observed (for technical information, see Furness and Hackney, 2006).

\section{Spectrin}

Postembedding immunogold labeling for spectrin was performed because it is a component of the cortical lattice and it is of interest to determine whether the lattice has any continuity with the cuticular plate or structures associated with the stereocilia and rootlets. Gold labeling shows that spectrin occurs throughout the cuticular plate in both rat (Fig. 9A) and guinea pig; we did not investigate this in mouse sections. In rat sections, there was a higher signal than in guinea pig; this was revealed in labeling of the stereocilia as well which was much weaker in guinea pig (Mahendrasingam et al., 1998). There was evidence of labeling along the upper and lateral edges of the cuticular plate. Rootlets and ankle regions of the stereocilia were also occasionally clearly labeled (Fig. $9 A, B)$. The lateral wall labeling, although present, was relatively weak.

\section{$\boldsymbol{\beta}$ - and $\boldsymbol{\gamma}$-actin}

We have shown previously in guinea pigs that rootlets contain both $\beta$ and $\gamma$ isoforms of actin (Furness et al., 2005). In rats, both isoforms were also detected and in similar distributions. In the apices of rat $\mathrm{OHCs}$, labeling for $\beta$-actin was observed in the stereocilia, cuticular plates, and rootlets, and in the junctions between hair cells and supporting cells, being stronger in the latter. The stereocilia (Fig. 9C) and the junctions at the edges of the cuticular plate appeared to be more strongly labeled than the main body of the cuticular plate, although denser patches in the latter corresponding to the more superficial meshwork described above were also prominently labeled (Fig. $9 C, D)$. Stereociliary labeling was twice as strong as that over the cuticular plates. The electron-dense core of the rootlets was strongly labeled compared with the surrounding lighter area and labeled rootlets were seen as with conventional transmission electron microscopy, extending beyond the cuticular plate into the cytoplasm (Fig. $9 C, D$ ). No significant labeling of the lateral wall was observed for $\beta$-actin.

In the apices of OHCs, labeling for $\gamma$-actin was observed in the stereocilia, cuticular plates, and rootlets, and in the junctions between the hair cells and supporting cells. Similar to $\beta$-actin, labeling was predominantly seen over the electron-dense core of the rootlet and the superficial meshwork (Fig. 9E,F). Rootlets that extend beyond the cuticular plate of the OHC contain both $\beta$ - and $\gamma$-actin isoforms, even in the portion extending into the cytoplasm (Fig. 9C,E). No difference was observed in the labeling density between the stereocilia and cuticular plate in the OHC. In 
addition to the cuticular plate and stereocilia, the lateral wall of $\mathrm{OHC}$ was also labeled for $\gamma$-actin and there appeared to be continuous labeling between the two regions (Fig. 9G).

\section{Tropomyosin}

Labeling for tropomyosin was observed throughout the cuticular plates of OHCs. Although the labeling for tropomyosin was sparse, it was enriched in the rootlets in both rat and guinea pig where it was observed predominantly on the electrondense core compared with the halo (Fig. $10 A, B)$. Measurement of the labeling density over the rootlet and sheath area compared with that of the cuticular plate showed an approximately ninefold enrichment over the former $(22.64 \pm 25.07$ particles $/ \mu \mathrm{m}^{2}$, mean $\left.\pm \mathrm{SD}\right)$ compared with the latter $\left(2.6 \pm 1.03\right.$ particles $\left./ \mu \mathrm{m}^{2}\right)$. Although the SD for rootlet labeling was high, probably because of the fact that, on occasions, rootlets will be contained entirely within the section and so cannot be labeled, this difference was found to be significant using a one-tailed paired $t$ test comparing the two in 11 sections $(p>$ $0.05)$. Relatively little labeling was observed in the stereocilia or any other regions of the hair cells. Mouse was not investigated for this protein.

\section{Espin}

Labeling for the cochlear espin isoform was observed in the stereocilia of rats but not in any other regions of the hair cells. Predominantly peripheral labeling of the stereocilia was observed. No espin was detected in the rootlets (Fig. 10C).

\section{Prestin}

C-terminus prestin labeling was detected exclusively in the lateral plasma membrane terminating just below the edge of the cuticular plate and the dense junctional complex in rat hair cells. No systematic labeling was detected over apical or periapical membranes (Fig. 10D).

\section{Discussion}

The vertebrate hair bundle is composed of stiff, actin-filled stereocilia. Experiments in frog (Howard and Ashmore, 1986) and turtle (Crawford and Fettiplace, 1985) found that the stiffness of the stereocilia changed with a "square power" as opposed to a linear relationship with height above the cuticular plate, consistent with the stereocilia pivoting at their tapered ankle during mechanical stimulation. These experiments have not yet been performed in mammals, but the passive stiffness of OHC hair bundles (Beurg et al. 2008) is comparable with that of turtle hair bundles of similar height (Ricci et al. 2002), suggesting similar mechanical behavior. The ankle region is thus likely to be a crit-
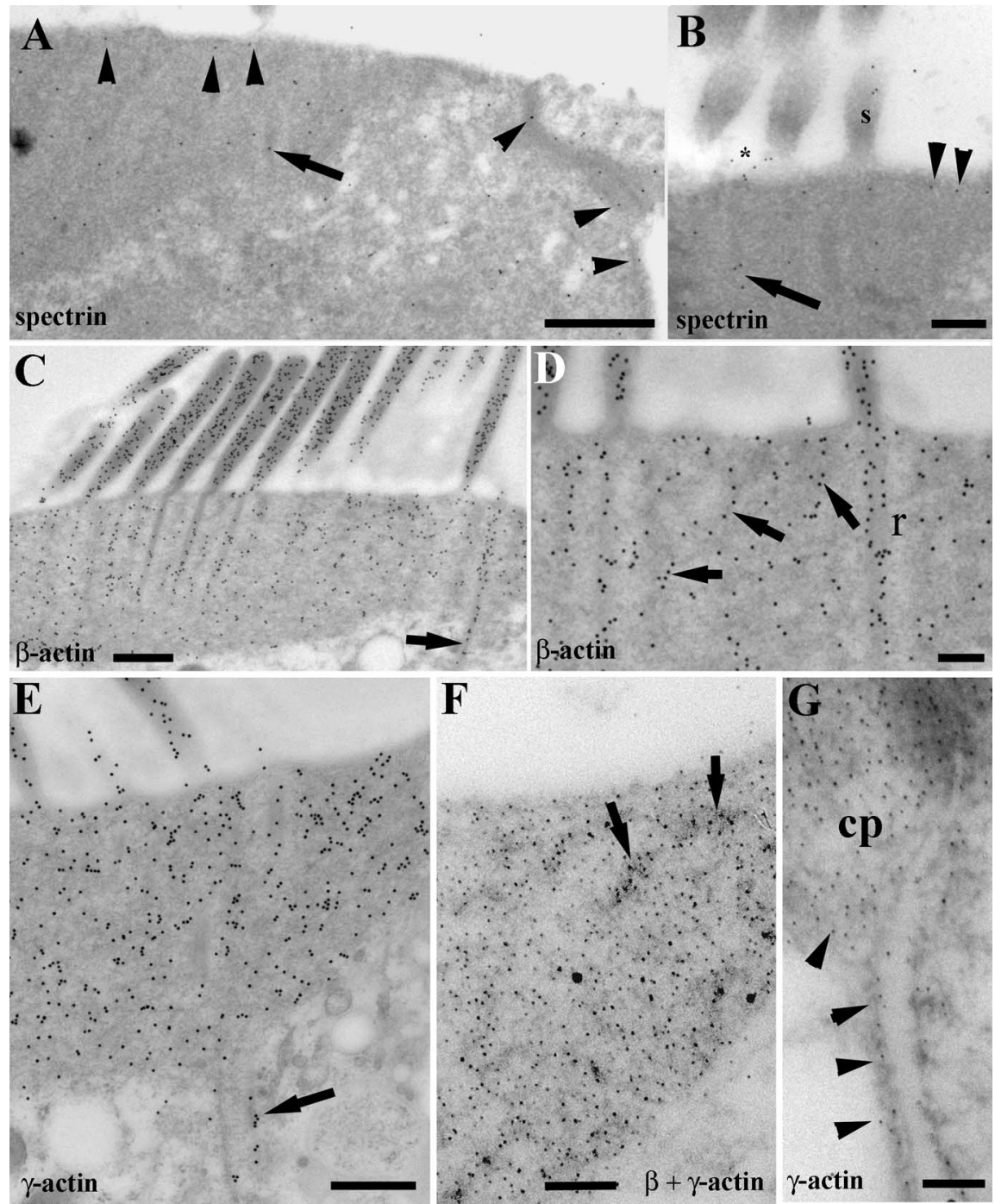

Figure 9. Immunogold labeling for spectrin, $\beta$-actin, and $\gamma$-actin. $\boldsymbol{A}-\boldsymbol{E}$, Rat $\mathrm{OHCs}$., $\boldsymbol{G}$, Guinea pig $\mathrm{OHCs}$. $\boldsymbol{A}$, Spectrin is distributed throughout the cuticular plate and is evident beneath the apical membrane and along the lateral margins (arrowheads). Labeling also occurs over the rootlets (arrow). $\boldsymbol{B}$, Spectrin labeling is evident not only in the subapical layer (arrowheads) and rootlets (arrow) but also in the ankle region (asterisk) and stereocilia (s). $\boldsymbol{C}, \beta$-Actin labeling is denser over the stereocilia and rootlets than the main matrix of the cuticular plate. A labeled rootlet extends below the cuticular plate (arrow). $D$, As well as prominent in the rootlets ( $r$ ), $\beta$-actin labeling is concentrated over the dark meshwork regions of the plate (arrows). $\boldsymbol{E}$, The labeling density for $\gamma$-actin is similar in the stereocilia and cuticular plate. Rootlets are also labeled, one extending deeper than meshwork (arrows). Large particles represent $\beta$-actin. $\mathbf{G}, \gamma$-Actin labeling is continuous from the cortical lattice (arrowheads) into the cuticular plate (ср). Scale bars: $\boldsymbol{A}, \boldsymbol{C}, \boldsymbol{E}, 400 \mathrm{~nm} ; \boldsymbol{B}, \boldsymbol{D}, 200 \mathrm{~nm} ; \boldsymbol{F}, \boldsymbol{G}, 100 \mathrm{~nm}$.

ical determinant of bundle stiffness and durability during prolonged stimulation.

The rootlets traverse the ankle region and have the appearance of anchoring structures. They are more conspicuous in mammalian cochlear hair cells where they are dense rods (Kimura, 1975; Itoh, 1982), than those of bird (Tilney et al., 1980) or turtle cochlea (Hackney et al., 1993). We have documented the dimensions and composition of the rootlets and examined how they vary with frequency location in the cochlea to provide further information about their possible functions. We hypothesized that they would display tonotopic gradients, such as changes in length, similar to those of stereociliary height along the organ of Corti.

The principal findings are that (1) in rodents rootlets consist of a dense central core thickened where the stereocilium joins the 


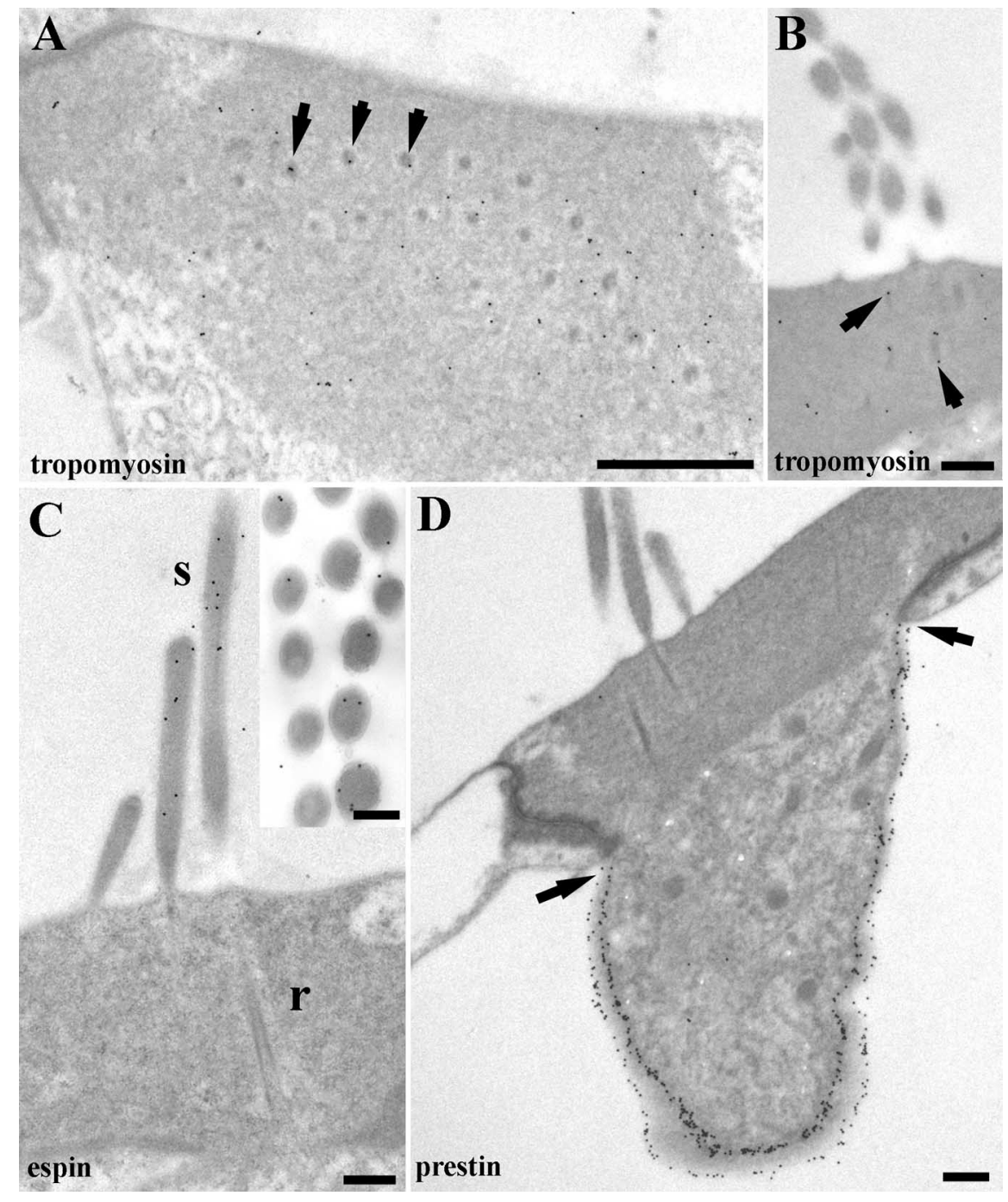

Figure 10. Labeling for tropomyosin, espin, and prestin in rat $\mathrm{OHCS.} A$, In horizontal sections, tropomyosin labeling is found throughout the cuticular plate but is frequently associated with rootlets (arrows). $\boldsymbol{B}$, Rootlet labeling for tropomyosin is more apparent in vertical sections (arrows). C, Labeling for espin is in the stereocilia (s) but is not detectable in the cuticular plate or rootlets ( $r$ ). Inset, Espin labeling tends to be more frequently observed near the periphery of the stereocilia. D, Prestin labeling is strong in the lateral wall but ends at the lower edge of the junctional region (arrows) and is completely absent from the apical membrane. Scale bars: $\boldsymbol{A}, 0.5 \mu \mathrm{m} ; \boldsymbol{B}, \boldsymbol{D}, 400 \mathrm{~nm} ; \boldsymbol{C}, 200 \mathrm{~nm}$.

apical surface of the cell, with peripheral dense filaments converging on the core in the stereociliary shaft; (2) their lengths in a given cochlear location are proportional to the stereociliary height, but change to a much smaller degree than the tonotopic changes in the latter. Our data on stereociliary height changes with cochleotopic location are in broad agreement with those published by Roth and Bruns (1992); (3) at the edges of the hair bundle in OHCs, rootlets contact the lateral wall at the junctional complex, and (4) the rootlet contains detectable amounts of spectrin, $\beta$ - and $\gamma$-actin, and tropomyosin, but not espin or prestin. The structural features of individual rootlets and how they attach to the lateral membrane are summarized in Figure 11.

The anatomical appearance of mammalian rootlets suggests that they support the stereocilia, assisting them to resist mechanical stresses experienced during acoustic stimulation. The relatively small tonotopic decrease in length relative to the stereociliary height lends support to this idea. The high frequency of mechanical vibrations (up to $60 \mathrm{kHz}$ in rats) experienced by mammalian basal hair cells implies that the ankles are subject to much more frequent mechanical stress and flexion compared with those in nonmammalian species (a few kilohertz), which may cause fatigue analogous to that in man-made materials. The rod structure may reflect greater durability compared with the more conical structure found in birds and turtles. The thickening of each rootlet at the ankle region is consistent with a strengthening role especially at the pivot point of the stereocilia. The presence of tropomyosin coating the actin filaments is likely to strengthen them (Isambert et al., 1995). In contrast, the absence of espin, and thus possibly of cross-linking would weaken the rootlets. The latter is also consistent with the closer packing of the actin filaments in guinea pig rootlets (Itoh, 1982). This region is a point of weakness because it tends to fracture when exposed to excessive acoustical stimulation (Liberman and Dodds, 1987), implying that a balance has to be struck between strength, durability, and flexibility.

Rootlet length may also reflect the distribution of the forces applied to different stereociliary rows. The tallest OHC stereocilia contact the overlying tectorial membrane (Lim, 1986) and are driven by displacement (Robles and Ruggero, 2001). The stimulus force is thus delivered to them and then transferred to the shorter stereocilia via cross links between them. It is thus possible that greater forces are experienced by the taller stereocilia than the shorter ones; hence, the former may require more support. Modeling of the type performed by Nam et al. (2006) may provide insight into this, but is beyond the scope of the present study.

It is uncertain to what extent the rootlets or ankles contribute to stiffness of the bundle. It has been argued, based on sequential treatments with BAPTA and protease to destroy different categories of extracellular cross links, that the ankle region contributes only a small fraction of bundle stiffness (Bashtanov et al., 2004). However, this estimate assumes that the bundle contains a simple parallel arrangement of lumped compliance which can substantially underestimate the ankle stiffness (Nam et al., 2006). Thus, it is possible that rootlets are more relevant to stiffness or anchoring in the mammalian cochlea than these measurements suggest. To assess this, additional data are needed to determine the extent to which the rootlets move within the cuticular plate.

It may be that the primary function of the extended rootlet into the cuticular plate is to prevent stereocilia from being pulled out of the apex of the hair cell during extreme deflections. Certainly this could be one reason why some rootlets appear to anchor to the lateral wall in the junctional complex of the lip (as illustrated schematically in Fig. 11), potentially providing a firm attachment that helps to withstand stereociliary damage. This arrangement may provide additional protection against the vertical stresses evoked during $\mathrm{OHC}$ somatic motility that are likely 
to be transmitted back to the tectorial membrane via the tall stereocilia. Rootlets may also help to control the content of the stereocilia; for example, mammalian stereocilia have a relative absence of diffusible calcium buffers compared with turtle ones (Hackney et al., 2003, 2005), which may suggest that the more substantive rootlets in the former form diffusion barriers that are not present in the latter.

However, although unlikely, it is possible that the connection to the lateral wall may provide a route by which somatic motility could influence bundle motility in cochlear amplification or vice versa. The latter would require that mechanical movements of the lateral membrane derived from prestin can be transmitted through the junctional complex; as yet, insufficient data are available about the junctional properties to refute or support this hypothesis. Continuity of the actin meshworks of the cuticular plate and cortical lattice could also provide a means for lateral wall motility to modulate bundle motion/ stiffness and vice versa via the rootlets. The presence of a dense apical layer over the cuticular plate containing spectrin and labeling for the latter along the junction suggests the possibility that the submembrane lattice continues in the absence of prestin throughout the apical region of the cell.

Understanding the composition of the rootlets is important in determining their role. Previous studies of a mouse model (Kitajiri et al., 2008) of the human deafness gene DFNB28 (Riazuddin et al., 2006) indicate that the rootlet contains an actin-associated protein, TRIOBP5, that may be involved in formation or maintenance of the rootlet. In adults, tropomyosin and unconventional myosins such as myosin VI around or within the rootlet region (Hasson et al., 1997) could provide a means of altering rootlet tension or stiffness. Furthermore, rootlet lengths can shorten within the stereociliary core after noise exposure potentially reducing the stiffness of the bundle (Liberman and Dodds, 1987). These features imply that the rootlets can be altered according to physiological needs. Hence, rootlet activity may be involved directly in modulating bundle properties.

\section{The cuticular plate meshwork}

A mesh of ordered filaments and associated dense material, which may correspond with $\alpha$-actinin-labeled structures described by Slepecky and Chamberlain (1985), surrounds the rootlets. $\alpha$-Actinin affects the viscoelastic properties of actin gels, making them stiffer than actin alone; such gels become more elastic in response to physical vibration by a probe of increasing frequency over 0.05-33 kHz (Wagner et al., 1999). Spectrin, an elastic molecule important for cell deformability (Salomao et al., 2006), is located in the rootlets and their vicinity; spectrin and $\alpha$-actinin in the meshwork provides a way to modulate the cuticular plate properties that could stiffen or loosen the gel around the rootlets. No structural differences were noted between apical and basal locations in this network but there could be different relative amounts of actin, spectrin and $\alpha$-actinin and therefore different frequency-related characteristics. The possible impor- tance of these structures in high-frequency hearing is suggested by the fact that in most nonmammalian vertebrates such as turtles the cuticular plate appears more homogeneous (Hackney et al., 1993).

\section{Conclusions}

Our data suggest that the rootlets give durability to and anchoring of stereocilia, but could also play a modulatory role in bundle mechanics. We also show that there are no substantial changes in rootlet dimensions with cochleotopic location despite the change in length of the stereocilia, but there is evidence for a physical connection between the rootlets and lateral wall that could increase the anchoring of the stereocilia. It is also possible that this connection and other features of the cytoskeleton in the apex might mediate interactions between bundle and somatic motility in the cochlear amplifier.

\section{References}

Bashtanov ME, Goodyear RJ, Richardson GP, Russell IJ (2004) The mechanical properties of chick (Gallus domesticus) sensory hair bundles: relative contributions of structures sensitive to calcium chelation and subtilisin treatment. J Physiol (Lond) 559:287-299.

Beurg M, Nam JH, Crawford A, Fettiplace R (2008) The actions of calcium on hair bundle mechanics in mammalian cochlear hair cells. Biophys J 94:2639-2653.

Boyd J, Risinger JI, Wiseman RW, Merrick BA, Selkirk JK, Barrett JC (1995) Regulation of microfilament organization and anchorage-independent growth by tropomyosin 1. Proc Natl Acad Sci USA 92:11534-11538.

Chan DK, Hudspeth AJ (2005a) Mechanical responses of the organ of Corti to acoustic and electrical stimulation in vitro. Biophys J 89:4382-4395.

Chan DK, Hudspeth AJ (2005b) $\mathrm{Ca}^{2+}$ current-driven nonlinear amplification by the mammalian cochlea in vitro. Nat Neurosci 8:149-155.

Crawford AC, Fettiplace R (1985) The mechanical properties of ciliary bundles of turtle cochlear hair cells. J Physiol (Lond) 364:359-379. 
Dallos P, Zheng J, Cheatham MA (2006) Prestin and the cochlear amplifier. J Physiol (Lond) 576:37-42.

Flock $\AA$, Flock B, Murray E (1977) Studies on the sensory hairs of receptor cells in the inner ear. Acta Otolaryngol 83:85-91.

Furness DN, Hackney CM (1985) Cross-links between stereocilia in the guinea pig cochlea. Hear Res 18:177-188.

Furness DN, Hackney CM (2006) The structure and composition of the stereociliary bundle of vertebrate hair cells. In: Springer handbook of auditory research (RA Eatock, R Fay, AN Popper, eds), pp 95-153. New York: Springer Science and Business Media.

Furness DN, Lehre KP (1997) Immunocytochemical localization of a highaffinity glutamate-aspartate transporter, GLAST, in the rat and guineapig cochlea. Eur J Neurosci 9:1961-1969.

Furness DN, Katori Y, Mahendrasingam S, Hackney CM (2005) Differential distribution of beta- and gamma-actin in guinea-pig cochlear sensory and supporting cells. Hear Res 207:22-34.

Gimona M, Vandekerckhove J, Goethals M, Herzog M, Lando Z, Small JV (1994) Beta-actin specific monoclonal antibody. Cell Motil Cytoskeleton 27:108-116.

Hackney CM, Fettiplace R, Furness DN (1993) The functional morphology of stereociliary bundles on turtle cochlear hair cells. Hear Res 69:163-175.

Hackney CM, Mahendrasingam S, Jones EM, Fettiplace R (2003) The distribution of calcium buffering proteins in the turtle cochlea. J Neurosci 23:4577-4589.

Hackney CM, Mahendrasingam S, Penn A, Fettiplace R (2005) The concentrations of calcium buffering proteins in mammalian cochlear hair cells. J Neurosci 25:7867-7875.

Hasson T, Gillespie PG, Garcia JA, MacDonald RB, Zhao Y, Yee AG, Mooseker MS, Corey DP (1997) Unconventional myosins in inner-ear sensory epithelia. J Cell Biol 137:1287-1307.

Hofer D, Ness W, Drenckhahn D (1997) Sorting of actin isoforms in chicken auditory hair cells. J Cell Sci 110:765-770.

Holley MC, Ashmore JF (1990) Spectrin, actin and the structure of the cortical lattice in mammalian cochlear outer hair cells. J Cell Sci 96:283-291.

Howard J, Ashmore JF (1986) Stiffness of sensory hair bundles in the sacculus of the frog. Hear Res 23:93-104.

Isambert H, Venier P, Maggs AC, Fattoum A, Kassab R, Pantaloni D, Carlier MF (1995) Flexibility of actin filaments derived from thermal fluctuations. Effect of bound nucleotide, phalloidin, and muscle regulatory proteins. J Biol Chem 270:11437-11444.

Itoh M (1982) Preservation and visualization of actin-containing filaments in the apical zone of cochlear sensory cells. Hear Res 6:277-289.

Jiang D, Furness DN, Hackney CM, Lopez DE (1993) Microslicing of the resin-embedded cochlea in comparison with the surface preparation technique for analysis of hair cell number and morphology. Br J Audiol 27:195-203.

Kennedy HJ, Crawford AC, Fettiplace R (2005) Force generation by mammalian hair bundles supports a role in cochlear amplification. Nature 433:880-883.

Kennedy HJ, Evans MG, Crawford AC, Fettiplace R (2006) Depolarization of cochlear outer hair cells evokes active hair bundle motion by two mechanisms. J Neurosci 26:2757-2766.
Kimura RS (1975) The ultrastructure of the organ of Corti. Int Rev Cytol 42:173-222.

Kitajiri S, Stepanyan R, Goodyear R, Richardson GP, Ahmed Z, Frolenkov G, Griffith A, Friedman T (2008) Genetic, cell biological and physiological analyses of a mouse model for DFNB28 deafness. ARO Abstr 31:496.

Liberman MC, Dodds LW (1987) Acute ultrastructural changes in acoustic trauma: serial-section reconstruction of stereocilia and cuticular plates. Hear Res 26:45-64.

Liberman MC, Zuo J, Guinan Jr JJ (2004) Otoacoustic emissions without somatic motility: can stereocilia mechanics drive the mammalian cochlea? J Acoust Soc Am 116:1649-1655.

Lim DJ (1986) Functional structure of the organ of Corti: a review. Hear Res 22:117-146.

Mahendrasingam S, Furness DN, Hackney CM (1998) Ultrastructural localisation of spectrin in sensory and supporting cells of guinea-pig organ of Corti. Hear Res 126:151-160.

Nam JH, Cotton JR, Peterson EH, Grant W (2006) Mechanical properties and consequences of stereocilia and extracellular links in vestibular hair bundles. Biophys J 90:2786-2795.

Riazuddin S, Khan SN, Ahmed ZM, Ghosh M, Caution K, Nazli S, Kabra M, Zafar AU, Chen K, Naz S, Antonellis A, Pavan WJ, Green ED, Wilcox ER, Friedman PL, Morell RJ, Riazuddin S, Friedman TB (2006) Mutations in TRIOBP, which encodes a putative cytoskeletal-organizing protein, are associated with nonsyndromic recessive deafness. Am J Hum Genet 78:137-143.

Ricci AJ, Crawford AC, Fettiplace R (2002) Mechanisms of active hair bundle motion in auditory hair cells. J Neurosci 22:44-52.

Robles L, Ruggero MA (2001) Mechanics of the mammalian cochlea. Physiol Rev 81:1305-1352.

Roth B, Bruns V (1992) Postnatal development of the rat organ of Corti. II. Hair cell receptors and their supporting elements. Anat Embryol 185:571-581.

Salomao M, An X, Guo X, Gratzer WB, Mohandas N, Baines AJ (2006) Mammalian alpha I-spectrin is a neofunctionalized polypeptide adapted to small highly deformable erythrocytes. Proc Natl Acad Sci USA 103:643-648.

Slepecky N, Chamberlain SC (1985) Immunoelectron microscopic and immunofluorescent localization of cytoskeletal and muscle-like contractile proteins in inner ear sensory hair cells. Hear Res 20:245-260.

Slepecky NB, Savage JE (1994) Expression of actin isoforms in the guinea pig organ of Corti: muscle isoforms are not detected. Hear Res 73:16-26.

Tilney LG, Derosier DJ, Mulroy MJ (1980) The organization of actin filaments in the stereocilia of cochlear hair cells. J Cell Biol 86:244-259.

Wagner O, Zinke J, Dancker P, Grill W, Bereiter-Hahn J (1999) Viscoelastic properties of $\mathrm{f}$-actin, microtubules, f-actin/alpha-actinin, and f-actin/ hexokinase determined in microliter volumes with a novel nondestructive method. Biophys J 76:2784-2796.

Zheng L, Sekerkova G, Vranich K, Tilney LG, Mugnaini E, Bartles JR (2000) The deaf jerker mouse has a mutation in the gene encoding the espin actin-bundling proteins of hair cell stereocilia and lacks espins. Cell 102: 377-385. 